\title{
Variational principle for some nonlinear problems
}

\author{
Yi $\operatorname{Tian}^{1,2}$
}

Received: 29 August 2021 / Accepted: 4 January 2022 / Published online: 4 February 2022

(c) The Author(s) 2022

\begin{abstract}
A variational principle is established by the semi-inverse method and used to solve approximately a nonlinear problem by the Ritz method. In this process, it may be difficult to solve a large system of algebraic equations, the Groebner bases theory (Buchberger's algorithm) is applied to solve this problem. The results show that the variational approach is much simpler and more efficient.
\end{abstract}

Keywords Variational principle $\cdot$ Semi-inverse method $\cdot$ Ritz method $\cdot$ Groebner bases

Mathematics Subject Classification 35A15 $\cdot 35 \mathrm{M} 12$

\section{Introduction}

With the rapid development of nonlinear science,various kinds of analytical methods were used to handle nonlinear problems, such as the homotopy perturbation method (He 1999, 2002, 2004, 2009, 2014; Anjum and He 2020a, b; Yu et al. 2019; Anjum et al. 2019; Ren et al. 2019; He and El-Dib 2020a,b, 2021), variational iteration method (He 1999, 2007, 2011, 2012a, b; He and Wu 2007; Anjum and He 2019; Liu et al. 2021), Taylor series method (He and Ji 2019; He 2019a, 2020a; He et al. 2020), Exp-function method ( $\mathrm{He}$ and $\mathrm{Wu} 2006$; He 2013; He et al. 2021), and variationalbased methods (He 2020b, 2021; He and Ain 2020; Wu 2021; Wang and Wei 2021). Recently, there have been some reasearch results in the field of differential equations, for example, in Wang et al. (2020), the ZIR of the fractal RC circuit are modeled by LFD, where the transient local fractional ordinary differential equation is obtained with aid of the law of switch and Kirchhoff Voltage Laws. In Cao and Dai (2021), Dai

$\bowtie$ Yi Tian

ttxsun@163.com

1 College of Data Science and Application, Inner Mongolia University of Technology, Hohhot 010080, China

2 Inner Mongolia Autonomous Region Engineering and Technology, Research Center of Big Data Based Software Service, Hohhot 010080, China 
and Wang (2020), Liu et al. (2020), Yu et al. (2020), Wu et al. (2020), new methods are presented to find traveling wave solutions of PDEs. In addition,the author has done some work on nonlinear problems, e.g. exact solutions (Tian 2019a, b, 2016) and numerical solutions (Tian 2018; Tian and Yan 2016) of integral and differential equations.

Each method has its advantages and disadvantages. For example, the Taylor series method is simple, but it is low convergence hinders its wide applications. The Expfunction method can lead to the analytical solutions, but its complex calculation makes those inaccessible who are not familiar some mathematics software. The variationalbased methods can obtain a globally valid solution, however, it is extremely difficult to establish a needed variational principle for a complex nonlinear problem, etc..

The variational principle play a important role in nonlinear science, especially for differential equations. For example, it can provides the conservation laws in an energy form, and reveals the possible solution structures of PDEs (He 2019b). In this paper, the semi-inverse method (He 2003a, b) is used to search variational principle of differential equations, then the Ritz method is effectively and conveniently used. In this process, it may be difficult to solve a large system of algebraic equations, the Groebner bases theory (Buchberger's algorithm) is applied to solve this problem.

The rest of this paper is organized as follows. In Sect. 2, we briefly review the basic Groebner Bases theory. In Sect. 3, Variational approach to some boundary value problems are studied. In Sect. 4, He's semi-inverse method is applied to solve some nonlinear problems. In Sect. 5, we conclude this paper and some discussions are also given there.

\section{Groebner Bases}

In the following, we list the basic results of Groebner Bases theory (Cox et al. 2007).

Definition 1 A subset $I \subset k\left[x_{1}, \ldots, x_{n}\right]$ is an ideal if it satisfies:

(i) $0 \in I$.

(ii) If $f, g \in I$, then $f+g \in I$.

(iii) If $f \in I$, and $h \in k\left[x_{1}, \ldots, x_{n}\right]$, then $h f \in I$.

Definition 2 Let $f_{1}, \ldots, f_{s}$ be polynomials in $k\left[x_{1}, \ldots, x_{n}\right]$. Then we set

$$
\left\langle f_{1}, \ldots, f_{s}\right\rangle=\left\{\sum_{i=1}^{s} h_{i} f_{i}: h_{1}, \ldots, h_{s} \in k\left[x_{1}, \ldots, x_{n}\right]\right\}
$$

The crucial fact is that $\left\langle f_{1}, \ldots, f_{s}\right\rangle$ is an ideal.

Definition 3 We will write $\bar{f}^{F}$ for the remainder on division of $f$ by the ordered stuple $F=\left(f_{1}, \ldots, f_{s}\right)$. If $F$ is a Groebner basis for $\left(f_{1}, \ldots, f_{s}\right)$, then we can regard $F$ as a set (without any particular order) by Proposition $I$. 
Definition 4 Let $I \subset k\left[x_{1}, \ldots, x_{n}\right]$ be an ideal. We will denote by $\mathbf{V}(I)$ the set

$$
\mathbf{V}(I)=\left\{\left(a_{1}, \ldots, a_{n}\right) \in k^{n}: f\left(a_{1}, \ldots, a_{n}\right)=0 \text { for all } f \in I\right\} .
$$

Definition 5 (Lexicographic Order) Let $\alpha=\left(\alpha_{1}, \ldots, \alpha_{n}\right)$ and $\beta=\left(\beta_{1}, \ldots, \beta_{n}\right) \in$ $Z_{\geq 0}^{n}$. We say $\alpha>_{\text {lex }} \beta$ if, in the vector difference $\alpha-\beta \in Z^{n}$, the leftmost nonzero entry is positive. we will write $x^{\alpha}>_{\text {lex }} x^{\beta}$ if $\alpha>_{\text {lex }} \beta$.

Theorem 1 (Buchberger's Algorithm) Let $I=\left\langle f_{1}, \ldots, f_{s}\right\rangle \neq 0$ be a polynomial ideal. Then a Groebner basis for I can be constructed in a finite number of steps by the following algorithm:

Input: $F=\left(f_{1}, \ldots, f_{s}\right)$

Output: a Groebner basis $G=\left(g_{1}, \ldots, g_{t}\right)$ for $I$, with $F \subset G$

$G:=F$

REPEAT

$$
G^{\prime}:=G
$$

FOR each pair $\{p, q\}, p \neq q$ in $G^{\prime} D O$

$$
S:=\overline{S(p, q)} G^{\prime}
$$

$U N T I L G=G^{\prime}$

$$
\text { IF } S: \neq 0 \text { THEN } G:=G \cup\{S\}
$$

\section{Variational approach to the boundary value problems}

\subsection{ODEs boundary value problems}

Example 1 Consider the following ODE (Lao 2015):

$$
y^{\prime \prime}+y=2 x
$$

with the boundary conditions

$$
y(0)=0, y(1)=0
$$

where $y^{\prime}$ is the differentiation with respect to $x$.

By the semi-inverse method, the variational principle of Eq. (1) reads

$$
J(y)=\int_{0}^{1}\left\{-\frac{1}{2}\left(y^{\prime}\right)^{2}+\frac{1}{2} y^{2}-2 x y\right\} d x,
$$

applying Ritz's method, we choose a trial function satisfying all the boundary conditions.

$$
\varphi_{n}=x^{n}(x-1)(n=1,2, \ldots)
$$


the approximate solution can be expressed as

$$
y_{n}(x)=\sum_{i=1}^{n} a_{i} \varphi_{i}=\sum_{i=1}^{n} a_{i} x^{i}(x-1) .
$$

Case 1. $n=1$

$$
y_{1}=\operatorname{ax}(x-1),
$$

where $a$ is a unknown constant to be further determined.

Substituting (6) into (3) yields

$$
J_{n=1}=\frac{a}{6}-\frac{3 a^{2}}{20}
$$

making $J_{n=1}$ stationary with respect to $a$ results in

$$
\frac{\partial J_{n=1}}{\partial a}=\frac{1}{6}-\frac{3 a}{10}=0
$$

we have $a=\frac{5}{9}$, and the first-order approximate solution of Eq. (1) is

$$
y_{1}=\frac{5}{9} x(x-1)
$$

Case 2. $n=2$.

$$
y_{2}=a x(x-1)+b x^{2}(x-1),
$$

where $a, b$ are unknown constants to be determined.

Substituting (10) into (3) yields

$$
J_{n=2}=-\frac{3 a^{2}}{20}-\frac{3 a b}{20}+\frac{a}{6}-\frac{13 b^{2}}{210}+\frac{b}{10},
$$

making $J_{n=2}$ stationary with respect to $a, b$ results in

$$
\begin{array}{r}
-\frac{3 a}{10}-\frac{3 b}{20}+\frac{1}{6}=0 \\
-\frac{3 a}{20}-\frac{13 b}{105}+\frac{1}{10}=0
\end{array}
$$

we have $a=\frac{142}{369}, b=\frac{14}{41}$, and the second-order approximate solution of Eq. (1) is

$$
y_{2}=\frac{142}{369} x(x-1)+\frac{14}{41} x^{2}(x-1) .
$$


Case 3. $n=3$.

$$
y_{1}=a x(x-1)+b x^{2}(x-1)+c x^{3}(x-1),
$$

where $a, b, c$ are unknown constants to be determined.

Substituting (15) into (3) yields

$$
J_{n=3}=-\frac{3 a^{2}}{20}-\frac{3 a b}{20}-\frac{19 a c}{210}+\frac{a}{6}-\frac{13 b^{2}}{210}-\frac{79 b c}{840}+\frac{b}{10}-\frac{103 c^{2}}{2520}+\frac{c}{15},
$$

making $J_{n=3}$ stationary with respect to $a, b, c$ results in

$$
\left\{\begin{array}{l}
-\frac{3 a}{10}-\frac{3 b}{20}-\frac{19 c}{210}+\frac{1}{6}=0 \\
-\frac{3 a}{20}-\frac{13 b}{105}-\frac{79 c}{840}+\frac{1}{10}=0 \\
-\frac{19 a}{210}-\frac{79 b}{840}-\frac{103 c}{1260}+\frac{1}{15}=0
\end{array}\right.
$$

Equation (17) sometimes is large and difficult to be solved by hand, even by software,such as MAPLE and MATLAB. In this paper,we use Buchberger's algorithm to solve this problem.

Let $I$ be the ideal

$$
\begin{aligned}
I= & \left\langle-\frac{3 a}{10}-\frac{3 b}{20}-\frac{19 c}{210}+\frac{1}{6},-\frac{3 a}{20}-\frac{13 b}{105}-\frac{79 c}{840}+\frac{1}{10},\right. \\
& \left.-\frac{19 a}{210}-\frac{79 b}{840}-\frac{103 c}{1260}+\frac{1}{15}\right\rangle \subset k[a, b, c],
\end{aligned}
$$

corresponding to the original system of Eq. (17), and we want to find all points in $\mathbf{V}(I)$.

Using Buchberger's algorithm with the lex order $a>b>c$, we find a Groebner basis:

$$
\begin{aligned}
& g_{1}=299 c+14, \\
& g_{2}=12259 b-4760, \\
& g_{3}=36777 a-13811,
\end{aligned}
$$

thus, there are three solutions altogether of $g_{1}=g_{2}=g_{3}=0$, which are

$$
a=\frac{13811}{36777}, b=\frac{4760}{12259}, c=-\frac{14}{299},
$$

since $\mathbf{V}(I)=\mathbf{V}\left(g_{1}, g_{2}, g_{3}\right)$,we have found all solutions of the original equations (17), and the third-order approximate solution of Eq. (1) is

$$
y_{3}=\frac{13811}{36777} x(x-1)+\frac{4760}{12259} x^{2}(x-1)-\frac{14}{299} x^{3}(x-1) .
$$


Example 2 Consider the following example (He 2003b):

$$
y^{(v i)}(x)=-6 e^{x}+y(x), 0<x<1,
$$

subject to the boundary conditions

$$
\begin{gathered}
y(0)=1, y^{\prime \prime}(0)=-1, y^{(i v)}(0)=-3, \\
y(1)=0, y^{\prime \prime}(1)=-2 e, y^{(i v)}(1)=-4 e .
\end{gathered}
$$

By the semi-inverse method,the variational principle of Eq. (24) reads

$$
J(y)=\int_{0}^{1}\left\{\frac{1}{2}\left(y^{\prime \prime \prime}\right)^{2}-6 e^{x} y+\frac{1}{2} y^{2}\right\} d x,
$$

applying Ritz's method, we choose a trial function

$$
\varphi_{n}=x^{n}(x-1)(n=1,2, \ldots)
$$

the approximate solution can be expressed as

$$
y_{n}(x)=\sum_{i=1}^{n} a_{i} \varphi_{i}=\sum_{i=1}^{n} a_{i} x^{i}(x-1) .
$$

Case 1. $n=1$.

$$
y_{1}=\operatorname{ax}(x-1),
$$

where $a$ is a unknown constant to be further determined.

Substituting (30) into (27) yields

$$
J_{n=1}=\frac{1}{60} a(a-360(e-3)),
$$

making $J_{n=1}$ stationary with respect to $a$ results in

$$
\frac{\partial J_{n=1}}{\partial a}=\frac{a}{60}+\frac{1}{60}(a-360(e-3))=0
$$

we have $a=180(e-3)$, and the first-order approximate solution of Eq. (24) is

$$
y_{1}=180(e-3) x(x-1) .
$$

Case 2. $n=2$.

$$
y_{2}=a x(x-1)+b x^{2}(x-1),
$$


where $a, b$ are unknown constants to be determined.

Substituting (34) into (27) yields

$$
J_{n=2}=\frac{1}{420}\left(7 a^{2}+7 a b-2520((e-3) a+(8-3 e) b)+7562 b^{2}\right),
$$

making $J_{n=2}$ stationary with respect to $a, b$ results in

$$
\begin{aligned}
\frac{1}{420}(14 a+7 b-2520(e-3)) & =0, \\
\frac{1}{420}(7 a+15124 b-2520(8-3 e)) & =0,
\end{aligned}
$$

we have

$$
a=\frac{360(15145 e-45428)}{30241}, b=-\frac{2520(7 e-19)}{30241},
$$

and the second-order approximate solution of Eq. (24) is

$$
y_{2}=\frac{360(15145 e-45428)}{30241} x(x-1)-\frac{2520(7 e-19)}{30241} x^{2}(x-1) \text {. }
$$

Case 3. $n=3$.

$$
y_{3}=a x(x-1)+b x^{2}(x-1)+c x^{3}(x-1),
$$

where $a, b, c$ are unknown constants to be determined.

Substituting (40) into (27) yields

$$
\begin{aligned}
J_{n=3}= & \frac{42 a^{2}-15120((e-3) a+(8-3 e) b+(11 e-30) c)}{2520} \\
& +\frac{42 a b+24 a c+45372 b^{2}+90735 b c+105845 c^{2}}{2520},
\end{aligned}
$$

making $J_{n=3}$ stationary with respect to $a, b, c$ results in

$$
\left\{\begin{array}{l}
\frac{84 a+42 b+24 c-15120(e-3)}{2520}=0, \\
\frac{42 a+90744 b+90735 c-15120(8-3 e)}{2520}=0, \\
\frac{24 a+90735 b+21160 c-15120(11 e-30)}{2520}=0,
\end{array}\right.
$$

Let $I$ be the ideal

$$
\begin{aligned}
I= & \left\langle\frac{84 a+42 b+24 c-15120(e-3)}{2520}, \frac{42 a+90744 b+90735 c-15120(8-3 e)}{2520},\right. \\
& \left.\frac{24 a+90735 b+211690 c-15120(11 e-30)}{2520}\right\rangle \subset k[a, b, c]
\end{aligned}
$$


corresponding to the system of Eq. (42).

Using Buchberger's algorithm with the lex order $a>b>c$, we find a Groebner basis:

$$
\begin{aligned}
& g_{1}=846721 c-1504440 e+4089960 \\
& g_{2}=-25605689761 b-60431928480 e+164225481840, \\
& g_{3}=-25605689761 a+4626241329780 e-13873846788900,
\end{aligned}
$$

thus,

$$
\begin{gathered}
\mathbf{V}(I)=\mathbf{V}\left(g_{1}, g_{2}, g_{3}\right)=\left\{a=\frac{1260(3671620103 e-11010989515)}{25605689761},\right. \\
\left.b=-\frac{5040(11990462 e-32584421)}{25605689761}, c=\frac{7560(199 e-541)}{846721}\right\}
\end{gathered}
$$

and the third-order approximate solution of Eq. (24) is

$$
\begin{aligned}
y_{3}= & \frac{1260(3671620103 e-11010989515)}{25605689761} x(x-1) \\
& -\frac{5040(11990462 e-32584421)}{25605689761} x^{2}(x-1)+\frac{7560(199 e-541)}{846721} x^{3}(x-1) .
\end{aligned}
$$

Remark 1 The solutions of Eq. (24) we obtained are not presented in (He 2003b).

\subsection{PDEs boundary value problems}

Example 3 Consider the following elliptic equation:

$$
-u_{x x}-u_{t t}+u=x^{2}+t^{2}, x \in[0,1] \times[0,1]
$$

subject to the boundary conditions

$$
u(x, 0)=u(x, 1)=u(0, t)=u(1, t)=0,
$$

By the semi-inverse method,the variational principle of Eq. (49) reads

$$
J(x, t)=\int_{0}^{1} \int_{0}^{1}\left\{\frac{1}{2} u_{x}^{2}+\frac{1}{2} u_{t}^{2}+\frac{1}{2} u^{2}-u\left(x^{2}+t^{2}\right)\right\} d x d t
$$

applying Ritz's method, we choose a trial function satisfying all the boundary conditions.

$$
\varphi_{n}=x^{n}(x-1) t(t-1)(n=1,2, \ldots),
$$


the approximate solution can be expressed as

$$
u_{n}(x, t)=\sum_{i=1}^{n} a_{i} \varphi_{i}=\sum_{i=1}^{n} a_{i} x^{i}(x-1) t(t-1) .
$$

Case 1. $n=1$.

$$
u_{1}=\operatorname{ax}(x-1) t(t-1),
$$

where $a$ is a unknown constant to be further determined.

Substituting (54) into (51) yields

$$
J_{n=1}=\frac{1}{600} a(7 a-10),
$$

making $J_{n=1}$ stationary with respect to $a$ results in

$$
\frac{\partial J_{n=1}}{\partial a}=\frac{7 a}{600}+\frac{1}{600}(7 a-10)=0,
$$

we have $a=\frac{5}{7}$, and the first-order approximate solution of Eq. (49) is

$$
u_{1}=\frac{5}{7} x(x-1) t(t-1)
$$

Case 2. $n=2$.

$$
u_{2}=a x(x-1) t(t-1)+b x^{2}(x-1) t(t-1)
$$

where $a, b$ are unknown constants to be determined.

Substituting (58) into (51) yields

$$
J_{n=2}=\frac{294 a^{2}+42 a(7 b-10)+5 b(20 b-49)}{25200}
$$

making $J_{n=2}$ stationary with respect to $a, b$ results in

$$
\begin{aligned}
\frac{588 a+42(7 b-10)}{25200} & =0, \\
\frac{294 a+100 b+5(20 b-49)}{25200} & =0,
\end{aligned}
$$

we have $a=\frac{285}{742}, b=\frac{35}{53}$, and the second-order approximate solution of Eq. (49) is

$$
u_{2}=\frac{285}{742} x(x-1) t(t-1)+\frac{35}{53} x^{2}(x-1) t(t-1) .
$$


Case 3. $n=3$.

$$
u_{3}=a x(x-1) t(t-1)+b x^{2}(x-1) t(t-1)+c x^{3}(x-1) t(t-1),
$$

where $a, b, c$ are unknown constants to be determined.

Substituting (63) into (51) yields

$$
J_{n=3}=\frac{882 a^{2}+6 a(147 b+86 c-210)+300 b^{2}+b(417 c-735)+163(c-3) c}{75600},
$$

making $J_{n=3}$ stationary with respect to $a, b, c$ results in

$$
\left\{\begin{array}{l}
\frac{1764 a+6(147 b+86 c-210)}{7560}=0 \\
\frac{882 a+600 b+417 c-735}{75600}=0 \\
\frac{516 a+417 b+163(c-3)+163 c}{75600}=0
\end{array}\right.
$$

Let $I$ be the ideal

$$
\begin{aligned}
I= & \left\langle\frac{1764 a+6(147 b+86 c-210)}{75600}, \frac{882 a+600 b+417 c-735}{75600},\right. \\
& \left.\frac{516 a+417 b+163(c-3)+163 c}{75600}\right\rangle \subset k[a, b, c],
\end{aligned}
$$

corresponding to the system of Eq. (65).

Using Buchberger's algorithm with the lex order $a>b>c$, we find a Groebner basis:

$$
\begin{aligned}
& g_{1}=787 c-756, \\
& g_{2}=41711 b+12523, \\
& g_{3}=83422 a-48669,
\end{aligned}
$$

thus,

$$
\mathbf{V}(I)=\mathbf{V}\left(g_{1}, g_{2}, g_{3}\right)=\left\{a=\frac{48669}{83422}, b=-\frac{12523}{41711}, c=\frac{756}{787}\right\},
$$

and the third-order approximate solution of Eq. (49) is

$$
u_{3}=\frac{48669}{83422} x(x-1) t(t-1)-\frac{12523}{41711} x^{2}(x-1) t(t-1)+\frac{756}{787} x^{3}(x-1) t(t-1) .
$$




\section{He's semi-inverse method}

For a given nonlinear partial differential equation

$$
R\left(u, u_{x}, u_{t}, u_{x x}, u_{x t}, u_{t t}, \ldots\right)=0
$$

the main steps of this method are as follows (Elboree 2015; Najafi and Arbabi 2016):

Step 1. seek solitary solutions of Eq. (72) by taking $u(x, t)=u(\xi), \xi=x-c t$, and transform Eq. (72) to the ordinary differential equation

$$
S\left(u, u^{\prime}, u^{\prime \prime}, \ldots\right)=0
$$

where prime denotes the derivative with respect to $\xi$.

Step 2. If possible,integrate Eq. (73) term by term one or more times, for simplicity, the integration constants set to zero.

Step 3. According to He's semi-inverse method, we construct the following trialfunctional

$$
J(u)=\int L d \xi
$$

where $L$ is a Lagrangian for Eq. (73).

Step 4. By the Ritz method, we can obtain different forms of solitary wave solutions, such as $u(\xi)=A \cdot \operatorname{sech}(B \xi), u(\xi)=A \cdot \operatorname{csch}(B \xi), u(\xi)=A \cdot \tanh (B \xi), u(\xi)=$ $A \cdot \operatorname{coth}(B \xi)$ and so on.In this paper, we search a solitary wave solution in the form

$$
u(\xi)=A \cdot \operatorname{sech}(B \xi),
$$

where $A$ and $B$ are constants to be further determined.

Substituting Eq. (75) into Eq. (74) and making $J$ stationary with respect to $A$ and $B$ results in

$$
\begin{gathered}
\frac{\partial J}{\partial A}=0, \\
\frac{\partial J}{\partial B}=0,
\end{gathered}
$$

solving simultaneously Eqs. (76) and (77), we obtain $A$ and $B$. Hence, the solitary wave solution Eq. (75) is well determined.

\subsection{The PHi-4 equation}

The PHi-4 equation play an important role in nuclear and particle physics over the decades. Let us consider the Phi-4 equation in the form (Akter and Akbar 2015):

$$
u_{t t}-u_{x x}+m^{2} u+\lambda u^{3}=0
$$


where $m$ and $\lambda$ are real valued constants.

Using the traveling wave variable $\xi=x-c t$, Eq. (78) is transformed into the following ODE:

$$
\left(c^{2}-1\right) u^{\prime \prime}+m^{2} u+\lambda u^{3}=0
$$

by He's semi-inverse method,we can obtain the following variational formulation:

$$
J(u)=\int_{0}^{\infty}\left[\frac{\left(1-c^{2}\right)}{2}\left(u^{\prime}\right)^{2}+\frac{m^{2}}{2} u^{2}+\frac{\lambda}{4} u^{4}\right] d \xi .
$$

Case A: we search for a soliton solution in the form

$$
u(\xi)=A \cdot \operatorname{sech}(\xi)
$$

by substituting (81) into (80), we obtain

$$
J=\frac{1}{6} A^{2}\left(A^{2} \lambda-c^{2}+3 m^{2}+1\right),
$$

to find the constant $A$,we need to solve the following equation:

$$
\frac{\partial J}{\partial A}=\frac{A^{3} \lambda}{3}+\frac{1}{3} A\left(A^{2} \lambda-c^{2}+3 m^{2}+1\right)=0,
$$

form Eq. (83),we obtain

$$
A= \pm \frac{\sqrt{c^{2}-3 m^{2}-1}}{\sqrt{2} \sqrt{\lambda}}
$$

therefore,the solitary wave solutions to the PHi-4 equations are constructed as follows:

$$
u(x, t)= \pm \frac{\sqrt{c^{2}-3 m^{2}-1}}{\sqrt{2} \sqrt{\lambda}} \cdot \operatorname{sech}(x-c t) .
$$

Case B: we search for a soliton solution in the form

$$
u(\xi)=A \cdot \operatorname{sech}^{2}(\xi)
$$

by substituting (86) into (80), we obtain

$$
J=\frac{1}{105} A^{2}\left(12 A^{2} \lambda-28 c^{2}+35 m^{2}+28\right),
$$

to find the constant $A$,we need to solve the following equation:

$$
\frac{\partial J}{\partial A}=\frac{8 A^{3} \lambda}{35}+\frac{2}{105} A\left(12 A^{2} \lambda-28 c^{2}+35 m^{2}+28\right)=0
$$


form Eq. (88), we obtain

$$
A= \pm \frac{\sqrt{\frac{7}{6}} \sqrt{4 c^{2}-5 m^{2}-4}}{2 \sqrt{\lambda}}
$$

therefore,the solitary wave solutions to the PHi-4 equations are:

$$
u(x, t)= \pm \frac{\sqrt{\frac{7}{6}} \sqrt{4 c^{2}-5 m^{2}-4}}{2 \sqrt{\lambda}} \cdot \operatorname{sech}^{2}(x-c t) .
$$

By a similar manipulation,we have:

$$
\begin{gathered}
u(x, t)= \pm \frac{\sqrt{\frac{33}{10}} \sqrt{9 c^{2}-7 m^{2}-9}}{4 \sqrt{\lambda}} \cdot \operatorname{sech}^{3}(x-c t), \\
u(x, t)= \pm \frac{\sqrt{\frac{143}{14}} \sqrt{16 c^{2}-9 m^{2}-16}}{8 \sqrt{\lambda}} \cdot \operatorname{sech}^{4}(x-c t), \\
u(x, t)= \pm \frac{\sqrt{\frac{4199}{14}} \sqrt{25 c^{2}-11 m^{2}-25}}{48 \sqrt{\lambda}} \cdot \operatorname{sech}^{5}(x-c t), \\
u(x, t)= \pm \frac{\sqrt{\frac{7429}{66}} \sqrt{36 c^{2}-13 m^{2}-36}}{32 \sqrt{\lambda}} \cdot \operatorname{sech}^{6}(x-c t),
\end{gathered}
$$

and so on.

Case C: we search for a soliton solution in the form

$$
u(\xi)=A \cdot \operatorname{sech}(\xi) \cdot \tanh (\xi),
$$

by substituting (95) into (80), we obtain

$$
J=\frac{1}{210} A^{2}\left(3 A^{2} \lambda-49 c^{2}+35 m^{2}+49\right),
$$

to find the constant $A$,we need to solve the following equation:

$$
\frac{\partial J}{\partial A}=\frac{A^{3} \lambda}{35}+\frac{1}{105} A\left(3 A^{2} \lambda-49 c^{2}+35 m^{2}+49\right)=0
$$

form Eq. (97),we obtain

$$
A= \pm \frac{\sqrt{\frac{7}{6}} \sqrt{7 c^{2}-5 m^{2}-7}}{\sqrt{\lambda}}
$$


therefore,the solitary wave solutions to the PHi-4 equations are:

$$
u(x, t)= \pm \frac{\sqrt{\frac{7}{6}} \sqrt{7 c^{2}-5 m^{2}-7}}{\sqrt{\lambda}} \cdot \operatorname{sech}(x-c t) \cdot \tanh (x-c t) .
$$

Remark 2 As far as the author know,the soliton solutions of Eq. (78) we obtained above are different from Akter and Akbar (2015).

\subsection{The conformable time-fractional Boussinesq equation}

Let us consider the time-fractional Boussinesq equation (Lakestani and Manafian 2018):

$$
D_{t}^{2 \alpha} u-u_{x x}-\left(u^{2}\right)_{x x}+u_{x x x x}=0,0<\alpha \leq 1
$$

Utilizing the wave transformation

$$
u(x, t)=u(\xi), \quad \xi=x-\frac{k t^{\alpha}}{\alpha}
$$

where $k \neq 0$. Substituting (101) into Eq. (100) yields a nonlinear ordinary differential equation,

$$
\left(k^{2}-1\right) u^{\prime \prime}-\left(u^{2}\right)^{\prime \prime}+u^{(4)}=0,
$$

where the prime indicates the derivation with respect to $\xi$. Integrating Eq. (102) twice and setting the constants of integration equal to zero, we have

$$
\left(k^{2}-1\right) u-u^{2}+u^{\prime \prime}=0
$$

by He's semi-inverse method,we can obtain the following variational formulation:

$$
J=\int_{0}^{\infty}\left[\frac{\left(k^{2}-1\right)}{2} u^{2}-\frac{1}{3} u^{3}-\frac{1}{2}\left(u^{\prime}\right)^{2}\right] d \xi
$$

Case A: we search for a soliton solution in the form

$$
u(\xi)=A \cdot \operatorname{sech}(\xi)
$$

by substituting (105) into (103), we obtain

$$
J=\frac{1}{12} A^{2}\left(\pi A-6 k^{2}+8\right)
$$


to find the constant $A$,we need to solve the following equation:

$$
\frac{\partial J}{\partial A}=-\frac{\pi A^{2}}{12}-\frac{1}{6} A\left(\pi A-6 k^{2}+8\right)=0,
$$

form Eq. (107),we obtain

$$
A=\frac{4\left(3 k^{2}-4\right)}{3 \pi}
$$

therefore,the solitary wave solutions is constructed as follows:

$$
u(\xi)=\frac{4\left(3 k^{2}-4\right)}{3 \pi} \cdot \operatorname{sech}(\xi)
$$

Case B: suppose soliton solution in the form

$$
u(\xi)=A \cdot \operatorname{sech}^{2}(\xi)
$$

by substituting (110) into (103), we obtain

$$
J=-\frac{1}{45} A^{2}\left(8 A-15 k^{2}+27\right)
$$

to find the constant $A$,we need to solve the following equation:

$$
\frac{\partial J}{\partial A}=-\frac{8 A^{2}}{45}-\frac{2}{45} A\left(8 A-15 k^{2}+27\right)=0
$$

form Eq. (112), we obtain

$$
A=\frac{1}{4}\left(5 k^{2}-9\right)
$$

therefore, the solitary wave solutions is:

$$
u(\xi)=\frac{1}{4}\left(5 k^{2}-9\right) \cdot \operatorname{sech}^{2}(\xi) .
$$


By a similar manipulation,we have:

$$
\begin{array}{r}
u(\xi)=\frac{2048\left(7 k^{2}-16\right)}{3675 \pi} \cdot \operatorname{sech}^{3}(\xi), \\
u(\xi)=\frac{11}{80}\left(9 k^{2}-25\right) \cdot \operatorname{sech}^{4}(\xi), \\
u(\xi)=\frac{524288\left(11 k^{2}-36\right)}{1486485 \pi} \cdot \operatorname{sech}^{5}(\xi), \\
u(\xi)=\frac{85}{896}\left(13 k^{2}-49\right) \cdot \operatorname{sech}^{6}(\xi),
\end{array}
$$

and so on.

Case C: we search for a soliton solution in the form

$$
u(\xi)=A \cdot \operatorname{sech}(\xi) \cdot \tanh (\xi),
$$

by substituting (119) into (103), we obtain

$$
J=-\frac{1}{90} A^{2}\left(4 A-15 k^{2}+36\right)
$$

to find the constant $A$, we need to solve the following equation:

$$
\frac{\partial J}{\partial A}=-\frac{2 A^{2}}{45}-\frac{1}{45} A\left(4 A-15 k^{2}+36\right)=0
$$

form Eq. (121), we obtain

$$
A=\frac{1}{2}\left(5 k^{2}-12\right)
$$

therefore, the solitary wave solution is:

$$
u(\xi)=\frac{1}{2}\left(5 k^{2}-12\right) \cdot \operatorname{sech}(\xi) \cdot \tanh (\xi) .
$$

\section{Conclusion}

In this paper,based on the semi-inverse method, variational formulation for some nonlinear problems are established, and the approximate solutions are easily obtained by the Ritz method. In this process, it may be difficult to solve a large system of algebraic equations, the Groebner bases theory (Buchberger's algorithm) is applied to solve this problem. At present, the application of Groebner bases theory maily on 
algebra polynomial ideals, in the next, we will study how to extend it to differential case.

\section{Declaration}

Conflict of interest The author declares no conflict of interests.

Open Access This article is licensed under a Creative Commons Attribution 4.0 International License, which permits use, sharing, adaptation, distribution and reproduction in any medium or format, as long as you give appropriate credit to the original author(s) and the source, provide a link to the Creative Commons licence, and indicate if changes were made. The images or other third party material in this article are included in the article's Creative Commons licence, unless indicated otherwise in a credit line to the material. If material is not included in the article's Creative Commons licence and your intended use is not permitted by statutory regulation or exceeds the permitted use, you will need to obtain permission directly from the copyright holder. To view a copy of this licence, visit http://creativecommons.org/licenses/by/4.0/.

\section{References}

Akter, J., Akbar, M.A.: Exact solutions to the Benney-Luke equation and the Phi-4 equations by using modified simple equation method. Results Phys. 5, 125-130 (2015)

Anjum, N., He, J.H.: Laplace transform: Making the variational iteration method easier. Applied Mathematics Letters 92, 134-138 (2019)

Anjum, N., He, J.H.: Higher-order homotopy perturbation method for conservative nonlinear oscillators generally and microelectromechanical systems' oscillators particularly. Int. J. Modern Phys. B 34(32), 2050313 (2020)

Anjum, N., He, J.H.: Homotopy perturbation method for N/MEMS oscillators. Math. Methods Appl. Sci. (2020). https://doi.org/10.1002/mma.6583

Anjum, N., Suleman, M., Lu, D.C., He, J.H., Ramzan, M.: Numerical iteration for nonlinear oscillators by Elzaki transform. J. Low Freq. Noise Vib. Act. Control 39(4), 879-884 (2019)

Cao, Q.H., Dai, C.Q.: Symmetric and anti-symmetric solitons of the fractional second- and third-order nonlinear schrodinger equation. Chin. Phys. Lett. 38(9), 090501 (2021)

Cox, D.A., Little, J., O'Shea, D.: Ideals, Varieties, and Algorithms: An Introduction to Computational Algebraic Geometry and Commutative Algebra, 3rd edn. Springer, New York (2007)

Dai, C.Q., Wang, Y.Y.: Coupled spatial periodic waves and solitons in the photovoltaic photorefractive crystals. Nonlinear Dyn. 102(3), 1733-1741 (2020)

Elboree, M.K.: Derivation of soliton solutions to nonlinear evolution equations using He's variational principle. Appl. Math. Modell. 39(14), 4196-4201 (2015)

He, J.H.: Homotopy perturbation technique. Comput. Methods Appl. Mech. Eng. 178(3-4), 257-262 (1999)

He, J.H.: Variational iteration method-a kind of non-linear analytical technique: Some examples. Int. J. Non-linear Mech. 34(4), 699-708 (1999)

He, J.H.: Homotopy perturbation method: a new nonlinear analytical technique. Appl. Math. Comput. 135(1), 73-79 (2002)

He, J.H.: Variational approach to the Thomas-Fermi equation. Appl. Math. Comput. 143(2-3), 533-535 (2003)

He, J.H.: Variational approach to the sixth-order boundary value problems. Appl. Math. Comput. 143(2), 537-538 (2003)

He, J.H.: Comparison of homotopy perturbation method and homotopy analysis method. Appl. Math. Comput. 156(2), 527-539 (2004)

He, J.H.: Variational iteration method-Some recent results and new interpretations. J. Comput. Appl. Math. 207(1), 3-17 (2007)

He, J.H.: An elementary introduction to the homotopy perturbation method. Comput. Math. Appl. 57(3), 410-412 (2009)

He, J.H.: A short remark on fractional variational iteration method. Phys. Lett. A 375(38), 3362-3364 (2011) 
He, J.H.: Notes on the optimal variational iteration method. Appl. Math. Lett. 25(10), 1579-1581 (2012)

He, J.H.: Asymptotic methods for solitary solutions and compactons. Abstract Appl. Anal. 2012, 916793 (2012)

He, J.H.: Exp-function method for fractional differential equations. Int. J. Nonlinear Sci. Numer. Simul. 14(6), 363-366 (2013)

He, J.H.: Homotopy perturbation method with two expanding parameters. Indian J. Phys. 88(2), 193-196 (2014)

He, J.H.: A simple approach to one-dimensional convection-diffusion equation and its fractional modification for E reaction arising in rotating disk electrodes. J. Electroanal. Chem. 854, 113565 (2019)

He, J.H.: Lagrange crisis and generalized variational principle for 3D unsteady flow. Int. J. Numer. Methods Heat Fluid Flow 30(3), 1189-1196 (2019)

He, J.H.: Taylor series solution for a third order boundary value problem arising in architectural engineering. Ain Shams Eng. J. 11(4), 1411-1414 (2020)

He, J.H.: Variational principle and periodic solution of the Kundu-Mukherjee-Naskar equation. Results Phys. 17, 103031 (2020)

He, J.H.: On the fractal variational principle for the telegraph equation. Fractals 29(1), 2150022 (2021)

He, J.H., Ain, Q.T.: New promises and future challenges of fractal calculus: From two-scale Thermodynamics to fractal variational principle. Thermal Sci. 24(2A), 659-681 (2020)

He, J.H., El-Dib, Y.O.: Periodic property of the time-fractional Kundu-Mukherjee-Naskar equation. Results Phys. 19, 103455 (2020)

He, J.H., El-Dib, Y.O.: Homotopy perturbation method for Fangzhu oscillator. J. Math. Chem. 58(10), 2245-2253 (2020)

He, J.H., El-Dib, Y.O.: The reducing rank method to solve third-order Duffing equation with the homotopy perturbation. Numer. Methods Partial Differ. Equ. 37(2), 1800-1808 (2021)

He, J.H., Ji, F.Y.: Taylor series solution for Lane-Emden equation. J. Math. Chem. 57(8), 1932-1934 (2019)

He, J.H., Wu, X.H.: Exp-function method for nonlinear wave equations. Chaos, Solitons and Fractals 30(3), 700-708 (2006)

He, J.H., Wu, X.H.: Variational iteration method: New development and applications. Comput. Math. Appl. 54(7-8), 881-894 (2007)

He, C.H., Shen, Y., Ji, F.Y., He, J.H.: Taylor series solution for fractal Bratu-type equation arising in electrospinning process. Fractals 28(1), 2050011 (2020)

He, J.H., Ji, F.Y., Mohammad-Sedighi, H.: Difference equation vs differential equation on different scales. Int. J. Numer. Methods Heat Fluid Flow 31(1), 391-401 (2021)

Lakestani, M., Manafian, J.: Analytical treatment of nonlinear conformable time-fractional Boussinesq equations by three integration methods. Opt. Quant. Electron. 50(4), 1-31 (2018)

Lao, D.Z.: Fundamentals of the Calculus of Variations, 3rd edn. National Defense Industry Press, BeiJing (2015).. ((in Chinese))

Liu, X.Y., Zhou, Q., Biswas, A., Alzahrani, A.K., Liu, W.J.: The similarities and differences of different plane solitons controlled by $(3+1)$-dimensional coupled variable coefficient system. J. Adv. Res. 24, 167-173 (2020)

Liu, Y.P., Wang, C.C., Li, S.J.: A fractal langmuir kinetic equation and its solution structure. Thermal Sci. 25(2B), 1351-1354 (2021)

Najafi, M., Arbabi, S.: Dark soliton and periodic wave solutions of the Biswas-Milovic equation. Optik 127(5), 2679-2682 (2016)

Ren, Z.F., Yao, S.W., He, J.H.: He's multiple scales method for nonlinear vibrations. J. Low Freq. Noise Vib. Act. Control 38(3-4), 1708-1712 (2019)

Tian, Y.: Exact solutions for a class of volterral integral-differential equations arising in viscoelastic fluid. Thermal Sci. 20(3), 807-812 (2016)

Tian, Y.: Markov chain Monte Carlo method to solve Fredholm integral equations. Thermal Sci. 22(4), 1673-1678 (2018)

Tian, Y.: Quasi hyperbolic function expansion method and tanh-function method for solving vibrating string equation and elastic rod equation. J. Low Freq. Noise Vib. Act. Control 38(3-4), 1455-1465 (2019)

Tian, Y.: Diffusion-convection equations and classical symmetry classification. Thermal Sci. 23(4), 21512156 (2019)

Tian, Y., Yan, Z.Z.: Monte Carlo method for solving a parabolic problem. Thermal Sci. 20(3), 933-937 (2016) 
Wang, K.L., Wei, C.F.: A powerful and simple frequency formula to nonlinear fractal oscillators. J. Low Freq. Noise Vib. Act. Control 40(3), 1373-1379 (2021)

Wang, K.J., Sun, H.C., Fei, Z.: The transient analysis for zero-input response of fractal RC circuit based on local fractional derivative. Alex. Eng. J. 59(6), 4669-4675 (2020)

Wu, Y.: Variational approach to fractal reaction-diffusion equations with fractal derivatives. Thermal Sci. 25(2B), 1425-1430 (2021)

Wu, G.Z., Yu, L.J., Wang, Y.Y.: Fractional optical solitons of the space-time fractional nonlinear Schrodinger equation. Optik 207, 164405 (2020)

Yu, D.N., He, J.H., Garcia, A.G.: Homotopy perturbation method with an auxiliary parameter for nonlinear oscillators. J. Low Freq. Noise Vib. Act. Control 38(3-4), 1540-1554 (2019)

Yu, L.J., Wu, G.Z., Wang, Y.Y., Chen, Y.X.: Traveling wave solutions constructed by Mittag-Leffler function of a $(2+1)$-dimensional space-time fractional NLS equation. Results Phys. 17, 103156 (2020)

Publisher's Note Springer Nature remains neutral with regard to jurisdictional claims in published maps and institutional affiliations. 